\title{
Fast diffusion mechanism of silicon tri-interstitial defects
}

\author{
Yaojun A. Du, ${ }^{1}$ Stephen A. Barr, ${ }^{2}$ Kaden R. A. Hazzard, ${ }^{3}$ Thomas J. Lenosky, ${ }^{1}$ Richard G. Hennig, ${ }^{1}$ and John W. Wilkins ${ }^{1}$ \\ ${ }^{1}$ Department of Physics, Ohio State University, Columbus, Ohio, USA \\ ${ }^{2}$ Department of Materials Science and Engineering, University of Illinois at Urbana-Champaign, Urbana, Illinois, USA \\ ${ }^{3}$ Department of Physics, Cornell University, Ithaca, New York, USA
}

(Received 12 April 2005; revised manuscript received 7 October 2005; published 15 December 2005)

\begin{abstract}
Molecular dynamics combined with the nudged elastic band method reveals the microscopic self-diffusion process of compact silicon tri-interstitials. Tight-binding molecular dynamics paired with ab initio density functional calculations speed the identification of diffusion mechanisms. The diffusion pathway can be visualized as a five defect-atom object both translating and rotating in a screwlike motion along $\langle 111\rangle$ directions. The density functional theory yields a diffusion constant of $4 \times 10^{-5} \exp \left(-0.49 \mathrm{eV} / k_{B} T\right) \mathrm{cm}^{2} / \mathrm{s}$. The low diffusion barrier of the compact tri-interstitial may be important in the growth of ion-implantation-induced extended interstitial defects.
\end{abstract}

DOI: 10.1103/PhysRevB.72.241306

PACS number(s): 61.72.Ji, 66.30.Lw, 71.15.Mb, 71.15.Pd

Following high-energy ion implantation, strongly supersaturated silicon self-interstitials can agglomerate to form macroscopic planar interstitial structures, $\{311\}$ defects. ${ }^{1}$ High-temperature annealing is necessary to remove lattice damage following ion implantation. However, during the annealing process the $\{311\}$ defects comprise a large reservoir of interstitials, ${ }^{2-4}$ which are released upon annealing and hence drive boron transient enhanced diffusion, ${ }^{5-7}$ an undesirable process which causes spatial broadening of boron concentration profiles. On the other hand, following lowenergy implantation, significant boron transient enhanced diffusion (TED) is still observed, even though no visible $\{311\}$ defects are developed. ${ }^{7}$

It is crucial to understand the diffusion of various point defects in order to have a quantitative understanding of boron TED. Measurements of the diffusion rate of defects in silicon have been reported in experiments. ${ }^{8-14}$ Current experimental techniques cannot cleanly resolve the diffusion rates of more complex defect species, and due to the atomicscale size of point defects, defect diffusion pathways cannot be resolved at all. ${ }^{15}$ Thus, numerical simulations provide a unique way to study the technologically important dynamics of point defects in silicon.

Previously, such simulations have studied numerous defect species using both classical and quantum Hamiltonians. ${ }^{16-20}$ Using nudged elastic band methods ${ }^{21}$ (NEB) within a density functional theory (DFT) code, Lopez et al. compute an activation energy of $0.28 \mathrm{eV}$ for a neutral single interstitial diffusing along the $X-T-X$ path. ${ }^{22} \mathrm{Kim}$ et al. estimates the self-diffusion barrier of a particular diinterstitial performing a reorientation to be $0.5 \mathrm{eV} .{ }^{17}$ Recent DFT-NEB calculations done in our group confirm this pathway but give a somewhat lower barrier of $0.3 \mathrm{eV} .^{23}$

Cogoni et al. ${ }^{24}$ use temperature-accelerated molecular dynamics ${ }^{25}$ with the Kwon et al. ${ }^{26}$ tight-binding (TB) potential to systematically study the diffusion of low-lying single interstitials, di-interstitials, and tri-interstitials. In particular they find all are fast diffusers with diffusion barriers of 0.94 , 0.89 , and $1.71 \mathrm{eV}$, respectively. We extend that work both with a more accurate tight-binding potential and with density-functional theory to further refine the diffusion path- way and the diffusion constant. Here we concentrate on the tri-interstitial finding an activation energy of $0.4-0.5 \mathrm{eV}$ in DFT (and $0.6 \mathrm{eV}$ in TB).

\section{Which tri-interstitial to study?}

In a previous work from our research group, ${ }^{27}$ the three lowest-energy tri-interstitial geometries, denoted $I_{3}^{a}, I_{3}^{b}$, and $I_{3}^{c}$, were identified by analysis of tight-binding molecular dynamics (MD) simulations followed by density functional relaxations. The TB calculations use the Lenosky et al. ${ }^{28}$ potential. $I_{3}^{a}$ is the ground state and $I_{3}^{b}$ and $I_{3}^{c}$ are excited states ${ }^{29}$ the density functional energies for the three defects in $216+3$ atom supercells were $2.24 \mathrm{eV} /$ atom, $2.37 \mathrm{eV} /$ atom, and $2.47 \mathrm{eV} /$ atom, respectively. The tightbinding MD simulations show only $I_{3}^{b}$ is a rapid diffuser, while $I_{3}^{a}$ and $I_{3}^{c}$ can be formed by interconversion of $I_{3}^{b}$, but are themselves immobile within the 5 ns time scale of the simulations. ${ }^{27}$ Hence, we focus on elucidating the microscopic diffusion process of the compact tri-interstitial $I_{3}^{b} . I_{3}^{b}$ has a compact defect geometry in which a perfect tetrahedron of four atoms replaces a single atom in the silicon lattice, with the faces oriented along the four symmetry related $\langle 111\rangle$ directions.

Tight-binding molecular dynamics determines an initial $I_{3}^{b}$ diffusion path. ${ }^{30}$ The density-functional theory uses the nudged elastic band method to refine the diffusion path and determines the accurate diffusion barrier. The self-diffusion of $I_{3}^{b}$ can be visualized in terms of the atoms most distorted during the process. In particular a five-atom object translates while rotating to avoid adjacent atoms. The NEB finds four equivalent paths along $\langle 111\rangle$ with a diffusion constant of $D$ $=4 \times 10^{-5} \exp \left(-0.49 \mathrm{eV} / k_{B} T\right) \mathrm{cm}^{2} / \mathrm{s}$.

\section{Minimum energy path and/or nudged elastic band}

Figures 1 (a) and 1(c) show two $I_{3}^{b}$ defects in neighboring sites that correspond to initial and final configurations along the diffusion path, the [111] direction. We obtain the initial pathway from analysis of molecular dynamics trajectories. ${ }^{27}$ 

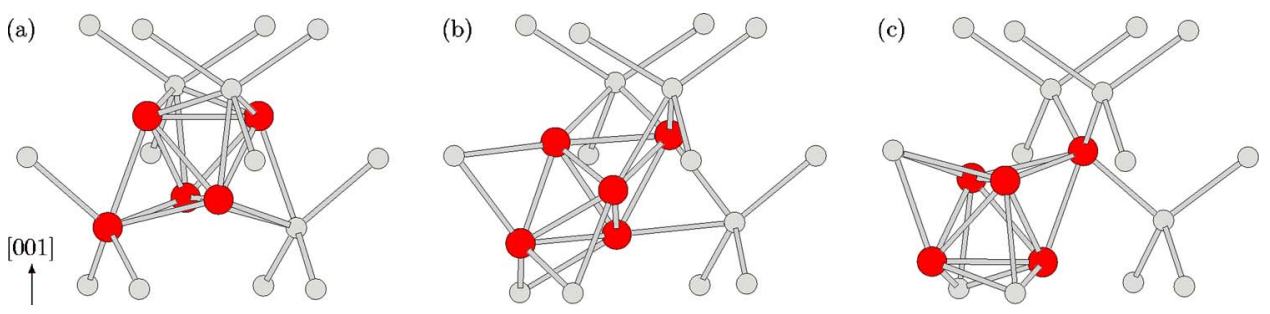

FIG. 1. (Color online) Diffusion of $I_{3}^{b}$ to the neighboring site along the $\langle 111\rangle$ direction. The initial, saddle, and final configurations are shown in (a), (b), and (c), respectively. Defect atoms are solid colored. The figures with more atoms can be found in Ref. 31.

The climbing-image NEB (CI-NEB) method ${ }^{32}$ refines an accurate pathway and finds the diffusion barrier between these two $I_{3}^{b}$ minima. The CI-NEB scheme guarantees that the image with the highest energy converges to the saddle point. All NEB calculations are performed with the Vienna Ab-initio Simulation Package (VASP) density functional code $\mathrm{c}^{33,34} \mathrm{em}-$ ploying the Perdew-Wang generalized gradient approximation (GGA) 35 and ultrasoft Vanderbilt-type pseudopotentials ${ }^{36}$ as provided by Kresse and Hafner. ${ }^{37}$ An initial relaxation of the two end points initiates a full relaxation of seven images along the path, keeping the volume of the cell fixed. Energy and atomic position convergence of $3 \mathrm{meV}$ and $0.005 \AA$, respectively, is confirmed for $64+3$ atom supercell by comparing the results for a $250 \mathrm{eV}$ energy cutoff and a $3 \times 3 \times 3 k$-point mesh with a $300 \mathrm{eV}$ cutoff and a $4 \times 4 \times 4$ mesh. The seven images are initialized by linear interpolation between the two relaxed end points. Each of the images is relaxed until the atomic forces are less than $10 \mathrm{meV} / \AA$.

Figure 2 shows the diffusion path with an activation energy of $0.49 \mathrm{eV}$. Harmonic transition state theory ${ }^{34}$ calculates the defect jump rate $\Gamma$ from the phonon frequencies at the minimum $\nu_{i}^{\min }$ and at the saddle point $\nu_{i}^{\text {sad }}$ and the activation energy $\Delta E$

$$
\Gamma=\Gamma_{0} e^{-\Delta E / k_{B} T}
$$

where the prefactor $\Gamma_{0}$ is given by

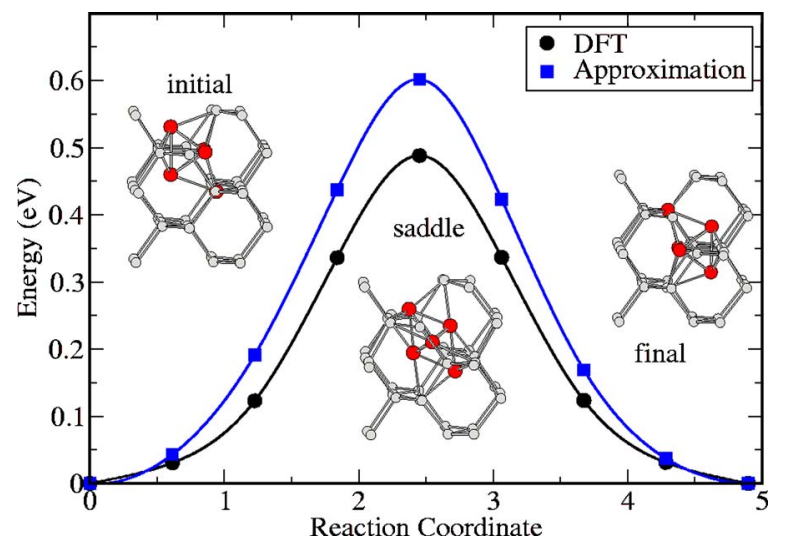

FIG. 2. (Color online) Transition paths of $I_{3}^{b}$ in DFT-GGA and the approximation of collective motion of five atoms within DFT-GGA.

$$
\Gamma_{0}=\prod_{i}^{3 N-3} \nu_{i}^{\min } / \prod_{i}^{3 N-4} \nu_{i}^{\text {sad }} .
$$

The dynamical matrix method within $a b$ initio GGA density functional theory determines the phonon frequencies. Each atom is displaced in the $\hat{x}, \hat{y}, \hat{z}$ direction by $0.03 \AA$, and the calculated forces are used to construct the Hessian matrix for the system, which is diagonalized to find the phonon frequencies. This yields a jump rate $\Gamma=0.2 \mathrm{THz} \exp (-0.49$ / $\left.k_{B} T\right)$. Calculations for a larger cell of $216+3$ atoms provide a diffusion barrier of $0.43 \mathrm{eV}$ estimating a finite size error of about $0.1 \mathrm{eV}$.

\section{Collective motion of atoms}

In the diffusion event, the five most-displaced atoms move collectively, with a screwlike motion. Figures 1(a), 1(b), and 1(c), showing the atomic configurations for initial, saddle, and final states, indicate significant displacement of the five solid colored atoms. All other atoms relax only slightly during the diffusion with a maximum displacement of $0.18 \AA$.

In order to illustrate the screwlike collective motion, we highlight the five highly displaced atoms in Fig. 3. Atoms $A$, $B$, and $C$ are located in a (111) plane, forming an equilateral triangle, and translate along and rotate, clockwise or counterclockwise, about the [111] axis defined by atoms $D$ and $E$. Meanwhile, atoms $D$ and $E$ translate along the same [111] direction. Atoms $D, A$, and $B$ form an equilateral triangle, whose normal vector indicates another diffusion direction, and all five atoms form a double tetrahedron. The bond between $A$ and $B$ is $2.49 \AA$ at the minimum, $6 \%$ longer than the perfect silicon-silicon bulk bond distance. During the diffu-

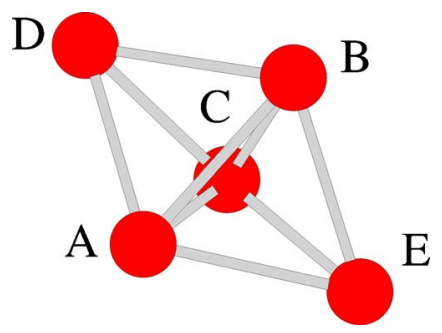

FIG. 3. (Color online) The five most displaced atoms during the diffusion. These five atoms move collectively. Atoms $D$ and $E$ define an axis of rotation ([111]), around which atoms $A, B$, and $C$ rotate clockwise or counterclockwise. 
TABLE I. The translation and rotation of five atoms along the [111] direction with respect to the initial state for all seven images in Fig 4.

\begin{tabular}{ccc}
\hline \hline Image & $\begin{array}{c}\text { Translation } \\
(\AA)\end{array}$ & $\begin{array}{c}\text { Rotation } \\
(\text { deg })\end{array}$ \\
\hline 1 & 0.18 & 0.4 \\
2 & 0.42 & 4.6 \\
3 & 0.57 & 16.4 \\
4 & 0.66 & 30.0 \\
5 & 0.75 & 43.6 \\
6 & 0.89 & 55.4 \\
7 & 1.13 & 59.6 \\
\hline \hline
\end{tabular}

sion event this bond length varies by less than $4.5 \%$. During defect migration the double-tetrahedron translates $1.31 \AA$ and rotates $60^{\circ}$ as shown in Fig. 1. Table I lists the values for the translation and rotation of the seven relaxed NEB images.

The diffusion event is approximated by a uniform translation and rotation of a fixed double tetrahedron. For instance, rotating the double tetrahedron of the initial configuration (image 1 ) by $16.4^{\circ}$, and translating it by $0.57 \AA$ while keeping its geometry fixed and relaxing the other atoms provides an approximation to image 3 . Applying this procedure to each of the images along the diffusion path, we obtain the approximate path in Fig. 2. The approximate activation energy is $0.6 \mathrm{eV}$, only $20 \%$ higher than the fully relaxed value.

Figure 4 shows the translation and rotation of the double tetrahedron during the diffusion. Translation occurs before the structure rotates. The double tetrahedron is displaced half way and rotated $30^{\circ}$ at the saddle point. The insets show images 3, 5 (saddle point) and 7 viewed from the [111] direction and illustrate that threefold symmetry persists during the diffusion.

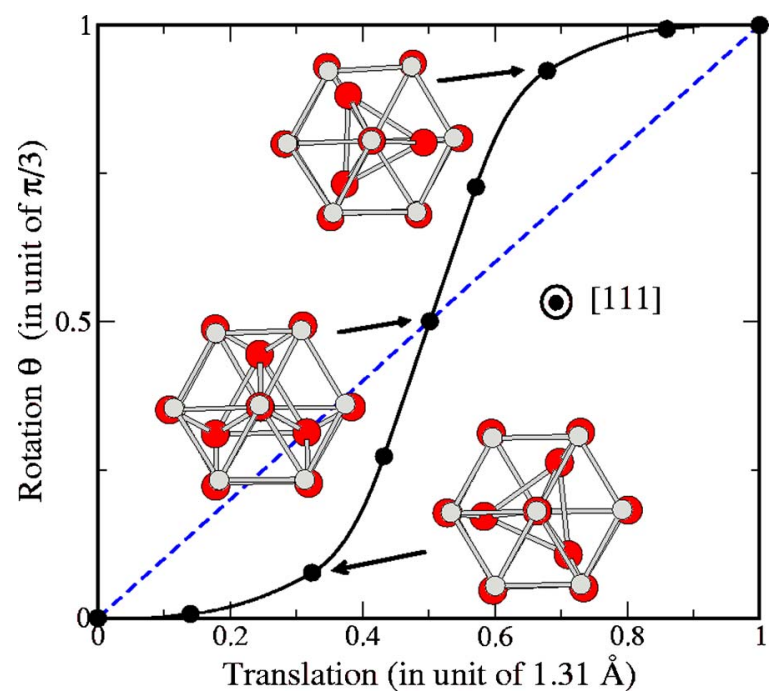

FIG. 4. (Color online) Transition path with seven images projected onto two-dimensional reduced coordinates. The inset shows images 3, 5 (saddle point), and 7 along the transition path.
TABLE II. Formation and activation energy of the neutral single-interstitial diffusing along the $X-T-X$ path, and of the neutral ground state di-interstitial and compact tri-interstitial during selfdiffusion. The formation energies are calculated in a $216+n$ atom supercell and the activation energies are calculated using NEB with a $64+n$ atom supercell. Details of the diffusion paths of single interstitials and di-interstitials will be published elsewhere (Ref. 23).

\begin{tabular}{ccc}
\hline \hline & $\begin{array}{c}\text { Formation energy } \\
\text { per atom } \\
(\mathrm{eV})\end{array}$ & $\begin{array}{c}\text { Activation } \\
\text { energy } \\
(\mathrm{eV})\end{array}$ \\
\hline$I_{1}$ & 3.810 & $0.29,0.28^{\mathrm{a}}$ \\
$I_{2}$ & 2.827 & 0.30 \\
$I_{3}^{b}$ & 2.368 & 0.49 \\
\hline \hline
\end{tabular}

$\overline{{ }^{a} \text { NEB result from Ref. } 22 \text { is calculated using } 216+1 \text { atom supercell. }}$.

\section{Diffusion rate}

The defect $I_{3}^{b}$ can move to four neighboring sites along the four symmetry equivalent $\langle 111\rangle$ directions. The diffusion constant $^{39}$ for this random walk is $D=2 \frac{4}{6} a^{2} \Gamma$, where the jump rate $\Gamma$ is defined by Eq. (1) and the displacement during the diffusion event is $a=1.31 \AA$. The resulting diffusion constant is $D=4 \times 10^{-5} \exp \left(-0.49 \mathrm{eV} / k_{B} T\right) \mathrm{cm}^{2} / \mathrm{s}$.

\section{Discussion}

Table II summarizes the $a b$ initio density functional theory results for formation and activation energy of $I_{1}, I_{2}$, and compact $I_{3}$ defects. The theoretical values of $0.28-0.49 \mathrm{eV}$ are considerably lower than the experimental results from metal-diffusion experiments which find activation energies, believed to be for single interstitials, typically ranging from $\sim 1.4$ to $1.8 \mathrm{eV}^{40}$ However, a recent experiment ${ }^{14}$ identifies enhanced diffusion of single interstitials at $150 \mathrm{~K}$, while noting it is inconsistent with results from earlier approaches. This lower temperature would be consistent with the NEB result for the energy barrier of single interstitial diffusion of $0.3 \mathrm{eV}$.

Our pathway for $I_{3}$ diffusion is the same as that found by Cogoni et al. ${ }^{24}$ in the $64+3$ atom supercell. On the other hand, our DFT barriers for $I_{1}, I_{2}$, and $I_{3}$ of $0.3,0.3$, and $0.4-0.5 \mathrm{eV}$ are much smaller than the tight-binding results by Cogoni et al. ${ }^{24}$ of $0.9,0.9$, and $1.7 \mathrm{eV}$. To understand the differences in the energy barriers between Cogoni's and our results, we calculate the diffusion path for $I_{3}^{b}$ with Kwon's TB potential ${ }^{26}$ using $\Gamma$ point and obtain a diffusion barrier of $1.2 \mathrm{eV}$ in contrast to the $0.6 \mathrm{eV}$ barrier with Lenosky's TB potential. ${ }^{28}$ We further check the $k$-point convergence using up to a $4^{3}$ mesh for both TB models. The converged diffusion barriers are $0.9 \mathrm{eV}$ and $0.6 \mathrm{eV}$ for Kwon's and Lenosky's TB potential, respectively. This indicates that the larger energy barriers of Cogoni et al. ${ }^{24}$ are due to the difference in the TB potential and insufficient $k$-point convergence.

We perform density of states (DOS) calculations for the interstitial defect along the diffusion path within a $216+3$ atom supercell. The DOS is calculated for the minimum, the 
saddle point, and an intermediate structure. The DOS shows a band gap of $0.71-0.74 \mathrm{eV}$ for all three configurations along the diffusion pathway with no states in the gap. ${ }^{41}$ The size of the gap is close to the band gap of pure Si in GGA of $0.72 \mathrm{eV}$. The lack of gap states indicates that charge transfer may not play a significant role for the diffusion of a compact tri-interstitial.

While the formation energy of $I_{3}$ is lower than $I_{1}$ on a per atom basis, it is much higher when considered on a total basis (see Table II). One implication that can be drawn from this is that under equilibrium conditions, $I_{3}$ is not present in any significant quantity, but under conditions which inject excess interstitials, ${ }^{5}$ such as ion implantation, it may be present in significant amounts due to the combination of mobile single interstitials. Our results have possible relevance for modeling transient enhanced diffusion which follows ion implantation. ${ }^{42}$

\section{Conclusions}

We have elucidated the pathway for the diffusion of the compact tri-interstitial $I_{3}^{b}$, the only fast diffusing tri- interstitial species in crystalline silicon. During the selfdiffusion event, five atoms move collectively in a screwlike motion along one of four symmetry-related $\langle 111\rangle$ directions. Our DFT result shows a low activation energy of $0.49 \mathrm{eV}$ and a diffusion constant of $4 \times 10^{-5} \exp \left(-0.49 \mathrm{eV} / k_{B} T\right)$ $\mathrm{cm}^{2} / \mathrm{s}$. Under conditions such as ion implantation that creates excess interstitials and hence favor cluster formation, $I_{3}^{b}$ diffusion may be an important process due to the low activation energy.

\section{Acknowledgments}

The work was supported in part by DOE-Basic Energy Sciences, Division of Materials Sciences (Contract No. DEFG02-99ER45795). Computing resources were provided by the Ohio Supercomputing Center. We gratefully acknowledge the Materials Computation Center, visitor program (K.H.), for helpful conversations with Jeongnim Kim.
${ }^{1}$ I. G. Salisbury and M. H. Loretto, Philos. Mag. A 39, 317 (1979).

${ }^{2}$ S. Takeda, Jpn. J. Appl. Phys., Part 2 30, L639 (1991).

${ }^{3}$ M. Kohyama and S. Takeda, Phys. Rev. B 46, 12305 (1992).

${ }^{4}$ J. Kim, J. W. Wilkins, F. S. Khan, and A. Canning, Phys. Rev. B 55, 16186 (1997).

${ }^{5}$ D. J. Eaglesham, P. A. Stolk, H.-J. Grossmann, and K. M. Poate, Appl. Phys. Lett. 65, 2305 (1994).

${ }^{6}$ A. Stolk et al., J. Appl. Phys. 81, 6031 (1997).

${ }^{7}$ L. H. Zhang, K. S. Jones, P. H. Chi, and D. S. Simons, Appl. Phys. Lett. 67, 2025 (1995).

${ }^{8}$ T. Y. Tan and U. Gösele, Appl. Phys. A 37, 1 (1985).

${ }^{9}$ H. Bracht, N. A. Stolwijk, and H. Mehrer, Phys. Rev. B 52, 16542 (1995).

${ }^{10}$ H. Zimmermann and H. Ryssel, Appl. Phys. A 55, 121 (1992).

${ }^{11}$ C. Boit, F. Lau, and R. Sittig, Appl. Phys. A 50, 197 (1990).

${ }^{12}$ G. B. Bronner and J. D. Plummer, J. Appl. Phys. 61, 5286 (1987).

${ }^{13}$ W. Wijaranakula, J. Appl. Phys. 67, 7624 (1990).

${ }^{14}$ P. Partyka et al., Phys. Rev. B 64, 235207 (2001).

${ }^{15}$ J. L. Benton, S. Libertino, P. Krigho, and D. J. Eaglesham, J. Appl. Phys. 82, 120 (1997).

${ }^{16}$ Y. Bar-Yam and J. D. Joannopoulos, Phys. Rev. Lett. 52, 1129 (1984).

${ }^{17}$ J. Kim et al., Phys. Rev. Lett. 83, 1990 (1999).

${ }^{18}$ S. K. Estreicher, M. Gharaibeh, P. A. Fedders, and P. Ordejon, Phys. Rev. Lett. 86, 1247 (2001).

${ }^{19}$ M. Gharaibeh, S. K. Estreicher, and P. A. Fedders, Physica B 308-310, 1 (2001).

${ }^{20}$ S. Birner et al., Solid State Commun. 120, 279 (2001).

${ }^{21}$ H. Jónsson, G. Mills, and K. W. Jacobsen, in Classical and Quantum Dynamics in Condensed Phase Simulations, edited by B. J. Berne, G. Ciccotti, and D. F. Coker (World Scientific, Singapore, 1998), p. 385.

${ }^{22}$ G. M. Lopez and V. Fiorentini, Phys. Rev. B 69, 155206 (2004).

${ }^{23}$ Yaojun A. Du et al. (unpublished).
${ }^{24}$ M. Cogoni, B. P. Uberuaga, A. F. Voter, and L. Colombo, Phys. Rev. B 71, 121203(R) (2005).

${ }^{25}$ M. R. Sorensen and A. F. Voter, J. Chem. Phys. 112, 9599 (2000).

${ }^{26}$ I. Kwon, R. Biswas, C. Z. Wang, K. M. Ho, and C. M. Soukoulis, Phys. Rev. B 49, 7242 (1994).

${ }^{27}$ D. A. Richie, J. Kim, S. A. Barr, K. R. A. Hazzard, R. Hennig, and J. W. Wilkins, Phys. Rev. Lett. 92, 045501 (2004).

${ }^{28}$ T. J. Lenosky et al., Phys. Rev. B 55, 1528 (1997).

${ }^{29}$ Our notation differs from that of Estreicher et al. (Ref. 18); our $I_{3}^{b}$ geometry corresponds to their $I_{3}^{a}$.

${ }^{30}$ The TB parameters (Ref. 28) were fit to an extensive DFT-derived database of mainly total energies and atomic forces for several geometries of low-energy structures.

${ }^{31}$ Yaojun A. Du et al.; cond-mat/0503479 (unpublished).

${ }^{32}$ G. Henkelman, B. P. Uberuaga, and H. Jónsson, J. Chem. Phys. 113, 9901 (2000).

${ }^{33}$ G. Kresse and J. Hafner, Phys. Rev. B 47, R558 (1993).

${ }^{34}$ G. Kresse and J. Furthmüller, Phys. Rev. B 54, 11169 (1996).

${ }^{35}$ J. P. Perdew, in Electronic Structure of Solids '91, edited by P. Ziesche and H. Eschrig (Akademie Verlag, Berlin, 1991), p. 11.

${ }^{36}$ D. Vanderbilt, Phys. Rev. B 41, R7892 (1990).

${ }^{37}$ G. Kresse and J. Hafner, J. Phys.: Condens. Matter 6, 8245 (1994).

${ }^{38}$ G. H. Vineyard, J. Phys. Chem. Solids 3, 121 (1957).

${ }^{39}$ A. R. Allnatt and A. B. Lidiard, Atomic Transport in Solids (Cambridge University Press, Cambridge, 1993), p. 272. In their Eq. (8.2.5), $z=4$, any $\left(s_{j, a}\right)^{2}=1 / 3$, and there is an additional sum over two rotation directions.

${ }^{40}$ H. Bracht, Proc.-Electrochem. Soc. 99, 357 (1999).

${ }^{41}$ The absence of defect levels in the gap during the $I_{3}^{b}$ diffusion may be caused by continuous bond switching.

${ }^{42}$ I. Martin-Bragado et al., Phys. Rev. B 68, 195204 (2003). 